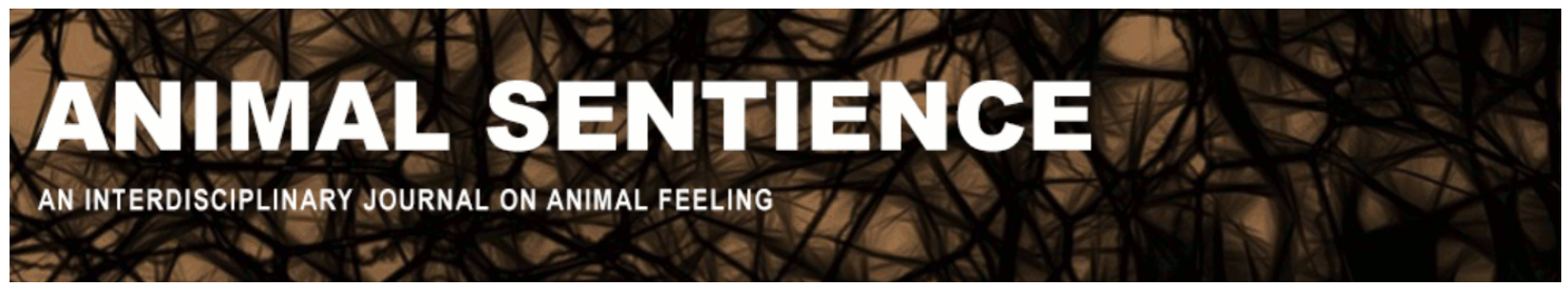

Seth, Anil K. and Dienes, Zoltan (2017) The value of Bayesian statistics for assessing credible evidence of animal sentience. Animal Sentience 16(22) DOI: $10.51291 / 2377-7478.1289$

Date of submission: 2018-01-02 Date of acceptance: 2018-01-03 (c) 


\title{
The value of Bayesian statistics for assessing credible evidence of animal sentience
}

Commentary on Birch on Precautionary Principle

\author{
Anil K. Seth and Zoltan Dienes \\ University of Sussex \\ Brighton, United Kingdom
}

\begin{abstract}
Determining what constitutes practically relevant, statistically significant evidence for animal sentience, under the precautionary principle, could be enhanced through Bayesian statistics. A Bayesian approach allows the incorporation of multiple evidence sources through prior probabilities, the tracking of changing evidence across time, and a principled means of adjusting evidentiary bars via Bayes factors.
\end{abstract}

Anil K. Seth is Professor of Cognitive and Computational Neuroscience and Co-Director of the Sackler Centre for Consciousness Science at the University of Sussex. He is also a Wellcome Trust Engagement Fellow and a Senior Fellow of the Canadian Institute for Advanced Research. www.anilseth.com

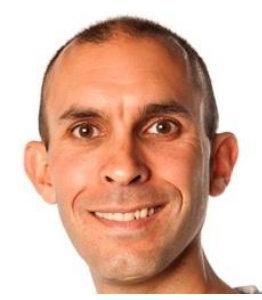

Zoltan Dienes is Professor of Psychology at the School of Psychology and the Sackler Centre for Consciousness Science at the University of Sussex. His main areas of publication, with over 150 papers, are consciousness and Bayesian statistics.

www.sussex.ac.uk/profiles/718

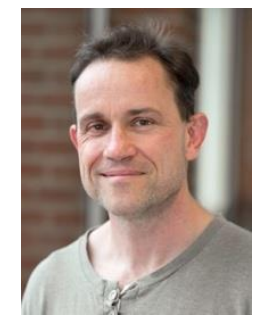

When and how should evidence for (or against) animal sentience drive new animal welfare policy? Birch (2017) deserves praise for his clear articulation of practical issues involved in this challenge under the "precautionary principle". We find his dual criteria of a (relatively) low evidentiary bar driving a subsequent action rule for welfare policy, both sensible and compelling. Put simply, Birch proposes that the relevant evidentiary bar is achieved when there is "sufficient evidence that animals of a particular order are sentient if there is statistically significant evidence, obtained by experiments that meet normal scientific standards, of the presence of at least one credible indicator of sentience in at least one species of that order".

Let's set aside issues of phylogenetic granularity (which Birch deals with rather nicely) and focus on the key challenge of deciding what constitutes statistically significant evidence under normal scientific standards. The examples Birch describes focus, unsurprisingly, on behavioural 
evidence relevant to putative aversive experience: self-administration of analgesia, conditioned place avoidance, and so on. Here, the actual evidence consists of behavioural observations under experimentally controlled conditions, supporting statistical inferences that will normally be based on frequentist analysis (the ubiquitous $p<0.05$ ). However, the relevant evidence for animal sentience may involve more than the kinds of behavioural data that can be submitted to such analyses. For example, identification of homologs or analogs of neuroanatomical structures or patterns of neural dynamics that are closely associated with sentience in humans (or in other creatures for which sentience is not in doubt, such as primates and perhaps all mammals), comprises valuable evidence in favour of sentience (Edelman, Baars, \& Seth, 2005; Edelman \& Seth, 2009; Seth, Baars, \& Edelman, 2005). How can such evidence be usefully incorporated into Birch's practical programme?

A natural way to accomplish this goal is to use Bayesian statistics, in which new evidence is considered in light of explicitly specified (probabilistic) prior beliefs. There are at least two senses in which this can be done, which we will summarise in turn. As a working example, consider an experiment designed to test whether a species $X$ shows conditioned place avoidance, a behaviour supposedly indicative of sentience (Sneddon, Elwood, Adamo, \& Leach, 2014).

First, Bayesian analysis can replace standard frequentist analysis (for example, of behavioural data). This is useful since Bayes factors (the Bayesian method of hypothesis testing) can indicate (quantitatively) the amount of evidence either against or in favour of the null hypothesis (e.g., that species $X$ does not show conditioned place avoidance) relative to the alternative, whereas standard frequentist statistics cannot provide evidence for the null hypothesis (Dienes, 2011). Thus, Bayesian statistics can tell us when the evidence is quantifiably insensitive, which can be useful for prompting additional data collection.

Second, Bayesian reasoning can quantify the strength of a particular behavioural finding as evidence for the higher-order hypothesis that species $\mathrm{X}$ is sentient. In this case, relevant prior beliefs can be shaped by additional (non-behavioural) sources of evidence. For example, one might take overall evidence to be stronger for sentience if conditioned place avoidance occurs in a species with a functional homolog of a mammalian thalamocortical network, or if it displays neural dynamics with high levels of dynamical complexity, and so forth (Edelman \& Seth, 2009). Most generally, behavioural evidence suggestive of sentience may be enough for species where there is a stronger prior belief for sentience (e.g., Octopus vulgaris) than for a species with prior belief favouring the opposite (e.g., Nautilus). Over time, the posterior belief (that species $X$ is sentient) can be continually updated as new evidence comes in.

One might immediately object that the validity of any inference like this will depend on the validity of the prior probability, and the whole point of a research programme in animal sentience is to move away from reliance on subjective prior judgements based on unreliable factors like anthropocentrism. These are important but misplaced concerns. The power of the Bayesian approach lies in (i) a principled incorporation of informed priors, such as those based on neuroanatomy and neurophysiology, and (ii) rendering all prior beliefs (both informed and highly subjective) explicit and transparent, and therefore available for community scrutiny. That is, the prior probability brought to bear for considering behavioural evidence should itself be based on evidence.

There is of course more to Bayes than this. Another advantage is that evidentiary bars can easily be adjusted by setting different Bayes factor thresholds, separately from adjusting priors. 
For example, if there is an independent imperative for considering fish pain to be important for policy (perhaps because of the large populations involved), then a lower Bayes factor could be used when deciding whether existing evidence is sufficiently strong to drive new policy. This usefully separates evaluating the strength of evidence for accepting (or rejecting) a particular hypothesis from the strength of evidence taken as sufficient to drive new actions.

Bayesian reasoning - a specific form of inference to the best explanation - is practical reasoning. Birch informally recognises the value of Bayesian reasoning when he says that subjective experience (e.g., pain experience) can be the "best explanation" for certain behavioural phenomena (p. 7). We suggest that its explicit adoption within his framework could substantially enhance its practical impact.

\section{Acknowledgements}

We are grateful to the Dr. Mortimer and Theresa Sackler Foundation, which supports the Sackler Centre for Consciousness Science.

\section{References}

Birch, J. (2017). Animal sentience and the precautionary principle. Animal Sentience 16(1). Dienes, Z. (2011). Bayesian versus orthodox statistics: which side are you on. Perspectives on Psychological Sciences, 6(3), 274-290.

Edelman, D. B., \& Seth, A. K. (2009). Animal consciousness: a synthetic approach. Trends in Neurosciences, 32(9), 476-484.

Edelman, D. B., Baars, B. J., \& Seth, A. K. (2005). Identifying the hallmarks of consciousness in non-mammalian species. Consciousness and Cognition, 14(1), 169-187.

Seth, A. K., Baars, B. J., \& Edelman, D. B. (2005). Criteria for consciousness in humans and other mammals. Consciousness and Cognition, 14(1), 119-139.

Sneddon, L. U., Elwood, R. W., Adamo, S. A., \& Leach, M. C. (2014). Defining and assessing animal pain. Animal Behaviour, 97, 201-212. 